\title{
Instalación Fotovoltaica: el escenario en educación superior del Aprendizaje Basado en Proyectos para alumnos de Energías
}

\author{
Photovoltaic installation: the scenario in higher education of the Project Based \\ Learning for Energy students
}

\author{
CASTILLO-ZARATE, Ma. Alicia $\dagger^{*}$
}

Universidad Tecnológica de Aguascalientes, Área Electromecánica industrial y energías. Blvd. Juan Pablo II \# 1302 Fracc. Ex Hacienda La Cantera, CP 20200, Aguascalientes Ags. México.

ID 1 ${ }^{\text {er }}$ Autor: Ma. Alicia, Castillo-Zarate / ORC ID: 0000-0003-0812-1125, CVU CONACYT ID: 710160

DOI: $10.35429 / J H S .2019 .10 .3 .1 .5$

Recibido 24 de Octubre, 2019, Aceptado, 28 de Noviembre, 2019

\begin{abstract}
Resumen
El presente artículo presenta una experiencia de aprendizaje centrada en el estudiante, desarrollada a través del Aprendizaje Basado en Proyectos y colaborativo. El objetivo primordial se enfoca a la adquisición de habilidades cognitivas y pragmáticas en una de las vertientes más comunes de aprovechamiento de energía solar, a decir, las instalaciones fotvoltaicas. Por ello se describe la experiencia de aprendizaje en el proceso de instalación de 194 paneles solares con el proyecto Instalación fotovoltaica en granja avícola de la comunidad de Tapias Viejas en Aguascalientes. Se utiliza la técnica de observación en las conductas de los alumnos, así como la solicitud de un Reporte escrito como producto del proyecto; en éste, los estudiantes manifiestan su opinión y aprendizajes, los cuales se han incluido literalmente en la sección de Resultados. Cabe resaltar la motivación y pro actividad que los estudiantes manifestaron durante el desarrollo del proyecto; de sus opiniones, destaca la importancia de metodologías de aprendizaje situados, que promuevan en el estudiante la oportunidad de aplicar conocimientos, desarrollar habilidades y fortalecer actitudes, necesarias para su formación profesional.
\end{abstract}

Aprendizaje, Proyectos, Fotovoltaico

\begin{abstract}
Resumen
This article presents a learning experience focused on the student, developed through project-based learning and collaborative learning. The primary objective is oriented to the acquisition of cognitive and pragmatic skills in one of the most common aspects of solar energy use, to say, the photovoltaic installations. Therefore, the learning experience is described in the process of installing 194 solar panels with the project Photovoltaic installation in a poultry farm in the community of Tapias Viejas in Aguascalientes. The observation technique is used in the behaviors of the students, as well as the request for a written report as a product of the project; in this, the students express their opinion and learnings, which have been included literally in the Results section. It is worth mentioning the motivation and pro activity that the students manifested during the development of the project; of their opinions, emphasizes the importance of situated learning methodologies, which promote in the student the opportunity to apply knowledge, develop skills and strengthen attitudes, necessary for their professional training.
\end{abstract}

Learning, Projects, Photovoltaic

Citación: CASTILLO-ZARATE, Ma. Alicia. Instalación Fotovoltaica: el escenario en educación superior del Aprendizaje Basado en Proyectos para alumnos de Energías. Revista de Educación Superior. 2019 3-10: 1-5

\footnotetext{
* Correspondencia del Autor (Correo electrónico: acastillo@utags.edu.mx)

$\dagger$ Investigador contribuyendo como primer autor
} 


\section{Introducción}

La puesta en práctica de estrategias de aprendizaje centradas en el estudiante, tales como el Aprendizaje Basado en Proyectos (ABP), el Trabajo Colaborativo y el proceso de aprendizaje significativo, implican el desarrollo de actividades con propósitos educativos específicos para la formación profesional, con el único interés de fortalecer y promover en el estudiante el logro del aprendizaje con sentido y utilidad (aprendizaje significativo) en y durante su formación.

La importancia de la inclusión de proyectos en el escenario educativo, estriba en que éstos surgen de una necesidad del entorno laboral (objetivo del proyecto) $\mathrm{y}$, al mismo tiempo durante su ejecución, le permiten al estudiante potenciar sus capacidades, desarrollar habilidades, reafirmar cualidades, alinear actitudes y atender debilidades, promoviendo en ellos el desarrollo y perfeccionamiento de sus competencias profesionales.

Este escenario de formación se traduce en aprendizajes, de los que derivan el progresivo desarrollo de habilidades técnicas que requiere el Técnico Superior Universitario en Energías Renovables área Solar, para realizar proyectos de generación de energía eléctrica a través de Instalaciones fotovoltaicas; por otro lado la estrategia de Aprendizaje basado en proyectos promueve en el alumno el continuo fortalecimiento de capacidades con la aplicación práctica de conocimientos adquiridos en el aula, y al mismo tiempo, permite el desarrollo de competencias actitudinales de empatía, tolerancia, trabajo en equipo, responsabilidad, liderazgo, el desarrollo de habilidades de su formación integral, principalmente las de interacción social y de comunicación, actitudes pertinentes a la demanda laboral de su perfil profesional; para lograrlo, la interacción de las estrategias de enseñanza-aprendizaje, los medios didácticos, el contexto social, las actividades de aprendizaje y los escenarios educativos, juegan un papel primordial.

La experiencia se coordinada por el docente de la materia Fisicoquímica Aplicada, y se realiza con la empresa Casa del Sol, responsable del proyecto.

La problemática que atiende este proyecto es el continuo incremento del consumo de energía eléctrica facturado a una granja avícola, el cual ha sido el detonante para que el dueño de la empresa haya solicitado un diagnóstico energético y la instalación de un sistema fotovoltaico interconectado a la red de CFE, para disminuir los costos por su consumo $\mathrm{y}$ al mismo tiempo que le permita generar energía eléctrica.

En esta circunstancia, la hipótesis central de esta propuesta para atender la problemática, supone que la puesta en marcha de un sistema fotovoltaico (SFV) interconectado a la red, de acuerdo a lineamientos especificados en estándar de competencia EC0586, disminuirá paulatinamente el costo, en la medida en que la generación de energía provea de este insumo a la granja y al mismo tiempo pueda abastecer a la red de Comisión Federal de Electricidad (CFE) compensando el consumo.

Bajo este contexto, este artículo muestra la experiencia de aprendizaje, con la descripción de las etapas de trabajo bajo el esquema de la estrategia Aprendizaje Basado en Proyectos, las cuales incluyen: Planeación de la experiencia, Desarrollo del proyecto, Ejecución del proyecto y Resultados.

\section{Metodologia a desarrollar}

El proyecto se desarrolla con base a la metodología del Aprendizaje Basado en proyectos, con las siguientes etapas:

a) Planeación de la experiencia

b) Desarrollo del proyecto

c) Ejecución del Proyecto

\section{A. Planeación de la experiencia}

Basado en el enfoque de Aprendizaje Basado en Proyectos, el punto de partida para el desarrollo y ejecución de este proyecto objeto de la presente intervención, responde a las siguientes interrogantes: 


\begin{tabular}{|c|c|c|}
\hline Pregunta & Respuesta & $\begin{array}{l}\text { Elemento del } \\
\text { proyecto }\end{array}$ \\
\hline $\begin{array}{l}\text { ¿Qué se quiere } \\
\text { hacer? }\end{array}$ & $\begin{array}{ll}\text { Realizar } & \text { Instalación } \\
\text { fotovoltaica } & \end{array}$ & Objetivo \\
\hline $\begin{array}{l}\text { ¿Por qué se } \\
\text { quiere hacer? }\end{array}$ & $\begin{array}{lll}\begin{array}{l}\text { Elevado } \\
\text { energía }\end{array} & \text { consumo } \\
\text { granja avícolaca } & \text { de } \\
\text { en } & \\
\end{array}$ & $\begin{array}{l}\text { Problema } \\
\text { situación } \\
\text { atender } \\
\end{array}$ \\
\hline $\begin{array}{l}\text { ¿Para qué se } \\
\text { quiere hacer? }\end{array}$ & $\begin{array}{l}\text { Para disminuir costos en } \\
\text { consumo de energía } \\
\text { eléctrica }\end{array}$ & $\begin{array}{l}\text { Justificación } \\
\text { impacto }\end{array}$ \\
\hline $\begin{array}{l}\text { ¿Dónde se quiere } \\
\text { hacer? }\end{array}$ & $\begin{array}{l}\text { En instalaciones de la } \\
\text { granja en Tapias Viejas } \\
\text { Aguascalientes }\end{array}$ & Lugar \\
\hline $\begin{array}{l}\text { ¿Cómo se va a } \\
\text { hacer? }\end{array}$ & $\begin{array}{l}\text { Con materiales de SFV, } \\
\text { de acuerdo a normas. }\end{array}$ & $\begin{array}{l}\text { Recursos } \\
\text { materiales }\end{array}$ \\
\hline $\begin{array}{l}\text { ¿Cuándo se va a } \\
\text { hacer? }\end{array}$ & $\begin{array}{l}\text { Actividades } \\
\text { programadas }\end{array}$ & $\begin{array}{l}\text { Cronograma } \\
\text { actividades }\end{array}$ \\
\hline $\begin{array}{ll}\text { ¿A quiénes se } \\
\text { dirige? }\end{array}$ & Granja avícola & Alcances \\
\hline $\begin{array}{l}\text { ¿Con quién se va } \\
\text { hacer? }\end{array}$ & Empresa Casa del Sol & $\begin{array}{l}\text { Recurso humano } \\
\text { responsable }\end{array}$ \\
\hline $\begin{array}{l}\text { ¿Quiénes lo van a } \\
\text { hacer? }\end{array}$ & $\begin{array}{l}\text { Alumnos y personal del } \\
\text { proveedor }\end{array}$ & $\begin{array}{l}\text { Recurso humano } \\
\text { colaborador }\end{array}$ \\
\hline
\end{tabular}

Tabla 1 Respuestas a interrogantes para la planeación del proyecto Instalación Fotovoltaica en Tapias Viejas Aguascalientes

Las respuestas a estas interrogantes definen los elementos del proyecto a desarrollar, mostrados en la tercera columna de la tabla.

\section{B. Desarrollo del proyecto}

Con la información de las respuestas, se define la siguiente estructura del proyecto:

Título: Instalación Fotovoltaica: el escenario en educación superior del Aprendizaje Basado en Proyectos para alumnos de Energías

Objetivo: Realizar instalación de sistema fotovoltaico interconectado a red, mediante conexión de paneles fotovoltaicos, cableado e inversores, con base a requerimientos de consumo y lineamientos especificados en estándar de competencia EC0586: Instalación de sistemas Fotovoltaicos en residencia, comercio e industria.

Problemática: El continuo incremento del costo de energía en una granja avícola, ha sido el detonante para que el dueño de la avícola solicite un diagnóstico energético, en cuyas propuestas, para eficientar el consumo y ahorro de energía se incluye la instalación de un sistema fotovoltaico interconectado a la red de CFE.

Interés de los alumnos: Participar en la Instalación del sistema fotovoltaico como práctica formativa en la Unidad 1. Energía Solar de la asignatura Fisicoquímica Aplicada del cuarto cuatrimestre del programa educativo de TSU en Energías Renovables Área Solar.
Justificación: La energía es la fuente principal para el bienestar y progreso de una sociedad; sin embargo su creciente demanda trae consigo principalmente las siguientes consecuencias: el incremento en la tarifa de consumo definido por los lineamientos indicados en la Reforma Energética y, el aumento en la contaminación atmosférica derivado de la quema de hidrocarburos (principal fuente convencional) para obtenerla.

En este escenario, el consumo de energía proveniente de fuentes alternas como la energía fotovoltaica asume mayor importancia, ya que aprovecha la energía del sol, no genera gases de efecto invernadero y, de acuerdo a la Reforma Energética, cuando el productor de esta energía satisface sus requerimientos, la producción excedente puede alimentar a la red de Comisión Federal de Electricidad, lo cual se traduce en disminución de costos de consumo de energía.

Responsable del proyecto: Empresa Casa del Sol, proveedor de la granja avícola y tutor de los alumnos para realizar la instalación

Recursos Humanos: Personal de Casa del Sol y alumnos de Energías

Recursos materiales y consumibles: Definidos en dimensionamiento energético como paneles solares, inversores, cableado, aislantes de neopreno, tornillos, terminales para los paneles.

Alcances: Abastecimiento de energía eléctrica a granja avícola $\mathrm{y}$, producción de energía eléctrica para inyectarse a la red de CFE.

\begin{tabular}{|c|c|c|c|c|c|c|c|c|c|}
\hline Etapas de & & & em & nas & e $S$ & pt & Dic 2 & 017 & \\
\hline Instalación & 4 & 5 & 6 & 7 & 8 & 9 & 10 & 11 & 12 \\
\hline Montaje de estructura & & & & & & & & & \\
\hline $\begin{array}{l}\text { Preparación } \\
\text { consumibles para la } \\
\text { instalación }\end{array}$ & & & & & & & & & \\
\hline Instalación de paneles & & & & & & & & & \\
\hline $\begin{array}{l}\text { Zanjas para colocar } \\
\text { registros y cables de } \\
\text { inversores }\end{array}$ & & & & & & & & & \\
\hline $\begin{array}{l}\text { Colocación de } \\
\text { estructuras para } \\
\text { montar inversores }\end{array}$ & & & & & & & & & \\
\hline $\begin{array}{l}\text { Conexión de paneles a } \\
\text { los inversores }\end{array}$ & & & & & & & & & \\
\hline $\begin{array}{l}\text { Conexión de cables de } \\
\text { inversores a línea de } \\
\text { CFE }\end{array}$ & & & & & & & & & \\
\hline $\begin{array}{l}\text { Puesta en marcha de } \\
\text { Instalación } \\
\text { fotovoltaica }\end{array}$ & & & & & & & & & \\
\hline
\end{tabular}

Tabla 2 Cronograma de actividades Fuente: Elaboración Propia

CASTILLO-ZARATE, Ma. Alicia. Instalación Fotovoltaica: el escenario en educación superior del Aprendizaje Basado en Proyectos para alumnos de Energías. Revista de Educación Superior. 2019 
C. Ejecución del proyecto (Desarrollo de la experiencia)

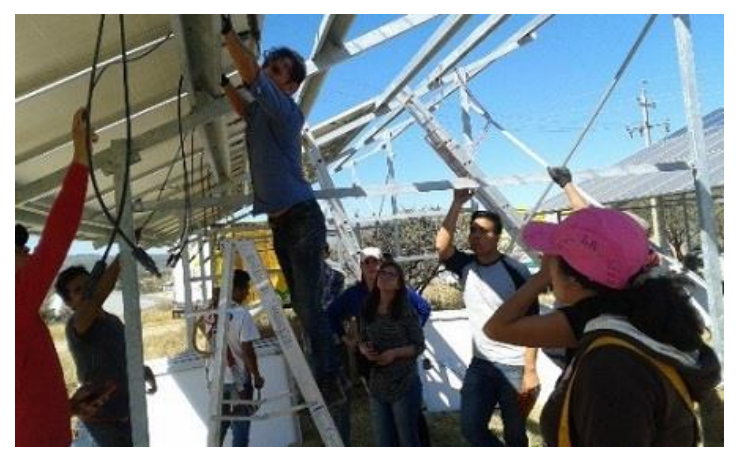

Figura 1 Demostración-ejecución de personal de la empresa Casa del Sol

Fuente: Elaboración Propia

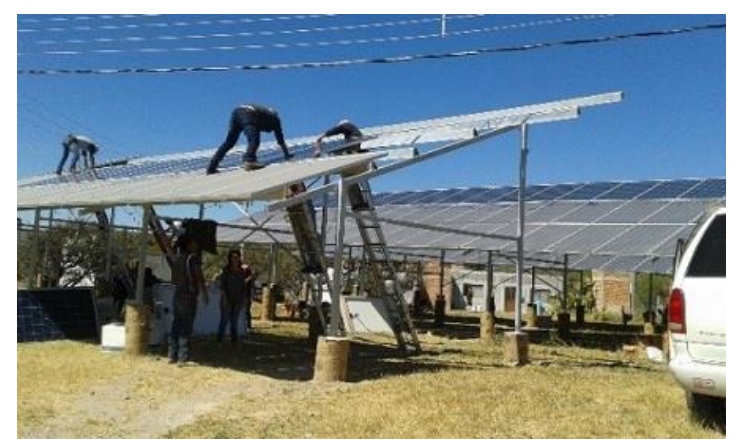

Figura 2 Instalación de paneles

Fuente: Elaboración Propia

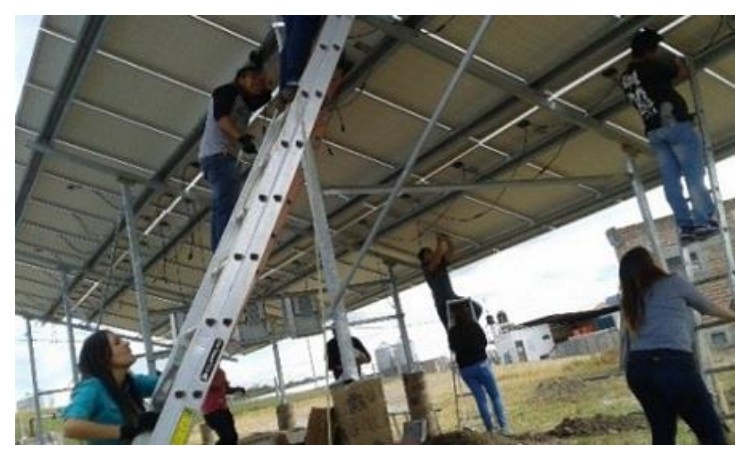

Figura 3 Conexión de paneles

Fuente: Elaboración Propia

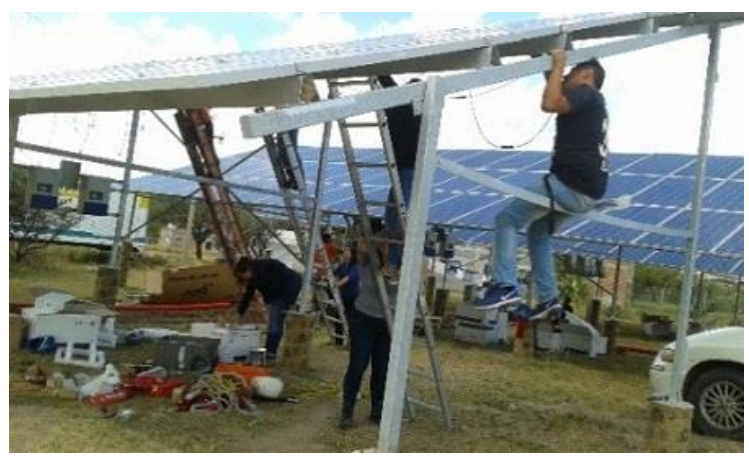

Figura 4 Cableado

Fuente: Elaboración Propia

\section{Resultados}

Considerando que el presente proyecto tiene como objetivo principal la adquisición de aprendizaje, traducido en habilidades cognitivas y pragmáticas a través del ABP, y derivado de las opiniones de los alumnos, se muestra a continuación algunos de sus aprendizajes obtenidos con esta experiencia:

- Montado de la estructura: Los alumnos identifican tipos y eficiencia de paneles de acuerdo a sus características, así como la cantidad de paneles que deben cargarse en una camioneta y la forma de trabajo en equipo para poder colocarlos en la estructura.

- Colocacación de paneles: Los alumnos aprenden cómo colocar los tornillos después del neopreno para que éstos queden fijos y se evite su caída, así mismo identifican que la razón por la que debe poner un material aislante es evitar una descarga por el contacto con la estructura.

- Conexiones En Serie: Los alumnos aprenden algunas medidas de seguridad durante la conexión, por ejemplo el uso de guantes y zapato con recubrimiento dieléctrico, lentes, chaleco de seguridad y casco en todo el tiempo de la instalación, para evitar algún accidente.

- Conexión a tierra en todos los paneles: Los alumnos aprenden la forma correctamente de colocar la tierra en todos los paneles con base a normas de seguridad, para evitr formar una antena y en caso de algún accidente no dañar el sistema.

- Preparación de zanjas para cableado: Los alumnos aprenden a usar herramienta apropiada para preparar zanjas desde el inversor de la instalación hacia el transformador, y colocar tubos PVC para introducir cableado de interconexión a la red.

- Ponchados: Los alumnos aprenden a realizar el ponchado de forma correcta, en el cable tipo hembra y macho para conectarlo al inversor. 
- Colocación de inversores y cajas de control: Los alumnos aprenden a usar el taladro para colocar inversores y cajas de control

- Conexión en inversores: Los alumnos aprenden a realizar conexiones trifásicas con inversores bifásicos en los inversores.

Cajas de control: Los alumnos realizan la instalación de cables en las cajas de control.

- Conexión a CD: Los alumnos aprenden a realizar la conexión de cables con corriente directa $(\mathrm{CD})$ hacia el inversor $\mathrm{y}$ la caja de control.

\section{Agradecimiento}

Se extiende un agradecimiento enorme por la oportunidad otorgada por el Ing. Raúl Coria de la empresa Casa del Sol, para colaborar en su empresa con este proyecto, y sobre todo por su valioso apoyo y asesoría técnica a los alumnos durante todas las etapas de esta instalación.

\section{Conclusiones}

$\checkmark$ El Aprendizaje Basado en Proyectos es una estrategia que permitió al alumno desarrollar la competencia para la instalación de sistemas fotovoltaicos en su contexto profesional.

$\checkmark$ El ABP situado en un contexto real, promovió la integración de aprendizajes cognitivos y desarrollo de habilidades, pero sobre todo la formación de actitudes y desarrollo de competencias sociales como la comunicación y la colaboración.

$\checkmark$ El desarrollo de actividades encaminadas al logro de un objetivo, bajo el esquema de ABP crea un ambiente de cooperación, apoyo y motivación para: el cumplimiento del proyecto y al mismo tiempo el aprendizaje que satisface los intereses de los alumnos.

\section{Recomendaciones}

$\checkmark$ Es importante que la función docente busque continua y permanentemente escenarios reales (aprendizajes situados) para el logro de aprendizajes significativos.
En lo sucesivo, la recomendación es planear prácticas de cada cuatrimestre, con proyectos reales de la región, para promover permanetemente el aprendizaje y desarrollo de competencias profesionales.

\section{Referencias}

Gonzalez Cabanach, R. (1997). Concepciones y enfoques de aprendizaje. Revista de Psicodidactica, 5-39.

Maldonado, P. M. (2008). Aprendizaje basado en proyectos colaborativos: Una experiencia en educación. Laurus. Revista de la Educacion, 158-180.

Rodriguez, P. M. (2010). La Teoria del Aprendizaje significativo en la perspectiva de la psicologia cognitiva. Barcelona, Espana: Octaedro. 